\title{
UQCRFS1N assembles mitochondrial respiratory complex-III into an asymmetric 21-subunit dimer
}

\section{Dear Editor,}

Mitochondrial respiratory chain consists of four multimeric protein complexes, Complex I-IV ( $\mathrm{Cl}$, NADH dehydrogenase; Cll, succinate:ubiquinone oxidoreductase; Clll, cytochrome $b c_{1}$ complex; and CIV, cytochrome $c$ oxidase). These four complexes transfer electrons from NADH or $\mathrm{FADH}_{2}$ to oxygen and pump protons from mitochondrial matrix to intermembrane space, generating electrochemical gradient across the inner membrane which is harnessed by complex $\mathrm{V}$ to synthesize ATP, providing the majority of energy acquired by living organisms. Respiratory chain complexes were reported to interact with each other to form supercomplexes, even megacomplex (Guo et al., 2017). However, despite decades of intensive research, many intriguing new findings concerning respiratory chain still emerging frequently in recent years (Baradaran et al., 2013; Guo et al., 2016; Schagger and Pfeiffer, 2000; Vinothkumar et al., 2014). CIII is the middle part of respiratory chain, transferring electrons from ubiquinone to cytochrome $\mathrm{c}$, and pumping protons in the meantime. All previous studies reported that $\mathrm{CIII}$ from vertebrate species was a homodimer consisting of 22 subunits in total (Fernandez-Vizarra and Zeviani, 2015, 2017), while after analysis of previous structural data, we draw a different conclusion.

The structures of the CIII homodimer from different species have been extensively studied, with the first structure of bovine CIII solved in 1997 by the Deisenhofer group (Xia et al., 1997). Since then, a total of 46 (23 bovine, 17 chicken, 2 porcine, 3 ovine, and 1 human) CIII structures from vertebrate species are available till now (Fernandez-Vizarra and Zeviani, 2017). In all these structures, CIII was descripted as a homodimer. However, unlike other subunits, the UQCRFS1N subunit in all these structures is poorly defined.
Although several structures have been solved with very high resolution, the full length $\mathrm{N}$-terminal processed peptide (178 amino acids, UQCRFS1N) of the iron-sulfur Rieske protein (UQCRFS1) subunit has not been assigned in all of these structures (Table 1). UQCRFS1N is the N-terminal mitochondrial targeting sequence of UQCRFS1, and after its cleavage from the precursor, this small peptide remains bound to CIII with unknown functions. In this letter, we show that one UQCRFS1N links the two 10-subunit CIII protomers together to form the intact CIII, which resultantly contains only 21 subunits rather than previously assumed 22 subunits (Fig. 1A and 1B).

Firstly, we rebuilt the high-resolution crystal structures of bovine CIII (PDB: 2A06) (Huang et al., 2005) and chicken CIII (PDB:3TGU) (Hao et al., 2012). To do this, we downloaded the bovine structure (PDB: 2A06), the chicken structure (PDB:3TGU) and their corresponding MTZ files. We deleted UQCRFS1N residues in those two models and refined them with their maps thus generating two meshes named mFo-DFc and 2mFo-DFc, colored with green and blue, respectively. Analysis was processed after modelling of UQCRFS1N back into the corresponding densities. Intriguingly, the $2.1 \AA$ density map of bovine CIII clearly shows that it contains two opposite orientations of the fulllength UQCRFS1N peptide (1-78 amino acids), with the $\mathrm{N}$ terminal binding in one CIII protomer and the $C$ terminal binding in the other CIII protomer (Fig. 1C-E). Both orientations can fit very well with the refined map, where the terminals of the two orientations occupy different densities while the middle regions of the two orientations share the same density. Refinement of the highest resolution chicken structure (PDB:3TGU) (Hao et al., 2012) gave same results (Fig. 1F). 
Table 1. Modelled UQCRFS1N residues in published CIII structures

\begin{tabular}{|c|c|c|c|c|}
\hline Species & PDB ID & Method & Modelled UQCRFS1N residues & Resolution $(\AA)$ \\
\hline Bos taurus & 1BE3 & X-ray & $46-78$ & 3.0 \\
\hline Bos taurus & $1 B G Y$ & X-ray & $46-78$ & 3.0 \\
\hline Bos taurus & $1 \mathrm{LOL}$ & X-ray & $1-57$ & 2.35 \\
\hline Bos taurus & $1 \mathrm{LON}$ & X-ray & $1-57$ & 2.6 \\
\hline Bos taurus & $1 \mathrm{NTK}$ & X-ray & $1-57$ & 2.6 \\
\hline Bos taurus & 1NTM & X-ray & $1-57$ & 2.4 \\
\hline Bos taurus & $1 \mathrm{NTZ}$ & X-ray & $1-57$ & 2.6 \\
\hline Bos taurus & $1 \mathrm{NU} 1$ & X-ray & $1-57$ & 3.2 \\
\hline Bos taurus & 1PP9 & X-ray & $32-78$ & 2.1 \\
\hline Bos taurus & 1PPJ & X-ray & $32-78$ & 2.1 \\
\hline Bos taurus & 1QCR & X-ray & $21-48$ & 2.7 \\
\hline Bos taurus & 1SQB & X-ray & $1-57$ & 2.69 \\
\hline Bos taurus & 1SQP & X-ray & $1-57$ & 2.7 \\
\hline Bos taurus & $1 S Q Q$ & X-ray & $1-57$ & 3.0 \\
\hline Bos taurus & $1 \mathrm{SQV}$ & X-ray & $1-57$ & 2.85 \\
\hline Bos taurus & $1 S Q X$ & X-ray & $1-57$ & 2.6 \\
\hline Bos taurus & $2 \mathrm{~A} 06$ & X-ray & $32-78$ & 2.1 \\
\hline Bos taurus & $2 F Y U$ & X-ray & $1-57$ & 2.26 \\
\hline Bos taurus & 4D6T & X-ray & $50-58,66-77,62-78$ & 3.57 \\
\hline Bos taurus & $4 \mathrm{D} 6 \mathrm{U}$ & X-ray & $50-63,66-78,62-78$ & 4.09 \\
\hline Bos taurus & $5 \mathrm{KLV}$ & X-ray & $20-29,46-64,66-70$ & 2.65 \\
\hline Bos taurus & 5 LUF & Electron microscopy & $46-78$ & 9.1 \\
\hline Bos taurus & $5 \mathrm{NMI}$ & X-ray & $49-78$ & 3.5 \\
\hline Gallus gallus & $1 \mathrm{BCC}$ & X-ray & - & 3.16 \\
\hline Gallus gallus & 2BCC & X-ray & - & 3.5 \\
\hline Gallus gallus & 3BCC & X-ray & - & 3.7 \\
\hline Gallus gallus & $3 C W B$ & X-ray & $47-77$ & 3.51 \\
\hline Gallus gallus & $3 \mathrm{H} 1 \mathrm{H}$ & X-ray & $47-77$ & 3.16 \\
\hline Gallus gallus & $3 \mathrm{H} 1 \mathrm{I}$ & X-ray & $47-77$ & 3.53 \\
\hline Gallus gallus & $3 \mathrm{H} 1 \mathrm{~J}$ & X-ray & $47-77$ & 3.0 \\
\hline Gallus gallus & $3 \mathrm{H} 1 \mathrm{~K}$ & X-ray & $47-77$ & 3.48 \\
\hline Gallus gallus & $3 \mathrm{H} 1 \mathrm{~L}$ & X-ray & $47-77$ & 3.21 \\
\hline Gallus gallus & $3 \mathrm{~L} 70$ & X-ray & $47-77$ & 2.75 \\
\hline Gallus gallus & 3L71 & X-ray & $47-77$ & 2.84 \\
\hline Gallus gallus & 3L72 & X-ray & $47-77$ & 3.06 \\
\hline Gallus gallus & 3L73 & X-ray & $47-77$ & 3.04 \\
\hline Gallus gallus & 3L74 & X-ray & $47-77$ & 2.76 \\
\hline Gallus gallus & 3L75 & X-ray & $47-77$ & 2.79 \\
\hline Gallus gallus & 3TGU & X-ray & $2-8,46-75$ & 2.7 \\
\hline Gallus gallus & $4 U 3 F$ & X-ray & $48-77$ & 3.23 \\
\hline Sus scrofa & $5 G P N$ & Electron microscopy & $46-78$ & 5.4 \\
\hline Sus scrofa & $5 G U P$ & Electron microscopy & $1-57$ & 4.0 \\
\hline Ovis aries & $5 \mathrm{~J} 4 \mathrm{Z}$ & Electron microscopy & $1-57$ & 5.8 \\
\hline
\end{tabular}


Table 1. continued

\begin{tabular}{lllll}
\hline Species & PDB ID & Method & Modelled UQCRFS1N residues & Resolution $(\AA)$ \\
\hline Ovis aries & 5 J7Y & Electron microscopy & $1-57$ & 6.7 \\
Ovis aries & $5 \mathrm{~J} 8 \mathrm{~K}$ & Electron microscopy & $1-57$ & 7.8 \\
Homo sapiens & $5 \mathrm{XTE}$ & Electron microscopy & $1-57$ & 3.4 \\
Homo sapiens & SXTE & Electron microscopy & $1-57$ & 3.4 \\
\hline
\end{tabular}

This composition is conserved in vertebrates. Unconventionally, we propose that a single UQCRFS1N molecule tethers the two 10-subuint CIII protomers, with the $\mathrm{N}$-terminal segment (residues 1-36) bound to one protomer and the C-terminal segment (residues 42-78) bound to the other. Furthermore, from the extra densities, we could build another UQCRFS1N molecule into CIII in a switched orientation, with the $\mathrm{N}$ - and $\mathrm{C}$-terminal segments of the peptide inserting into the opposite CIII protomers (Fig. 1A and 1B). The two UQCRFS1N molecules partially overlap with each other, indicating that they could not simultaneously exist in the same complex. Thus, they likely represent alternative conformations of CIII. Thus, each CIII complex has 21 subunits. Twenty subunits (each protomer containing ten subunits) form a symmetric dimer while the UQCRFS1N peptide inserts into the two protomers with two alternative orientations to assemble them together (Fig. $1 \mathrm{~A}$ and $1 \mathrm{~B}$ ).

The unusual binding mode of the UQCRFS1N peptide in CIII has not been previously observed. We checked 29 mammalian CIII (23 from bovine, 2 from porcine, 3 from ovine and 1 from human) and 17 chicken CIII structures available in the RCSB PDB protein data bank (https://www. rcsb.org), and found that none of them contains the fulllength UQCRFS1N peptide (Table 1), consistent with a previous report (Fernandez-Vizarra and Zeviani, 2017). All previous structural studies on CIII suggested that this complex was a symmetric homodimer. However, our refinement of bovine and chicken structures (PDB: 2A06 and 3TGU) indicates that a single UQCRFS1N peptide can insert into the two CIII protomers with two different orientations when
CIII is assembled into the respirasome (Fig. 1A and 1B). This further suggests that $\mathrm{CIII}$ has no preferred orientation when it binds to $\mathrm{Cl}$ and $\mathrm{CIV}$ to form the respirasome (Gu et al., 2016; Wu et al., 2016). Re-refinement of the previous high-resolution X-ray bovine and chicken CIII structures (PDB: 2A06 and 3TGU) suggests that a single UQCRFS1N peptide in two opposite orientations can tether the two CIII protomers together (Fig. 1A and 1B), even in the absence of $\mathrm{Cl}$ and CIV. This phenomenon is further consistent with our recent findings that both protomers of CIII have the abilities to bind with $\mathrm{Cl}$ to form the megacomplex- $\mathrm{I}_{2} \mathrm{III}_{2} \mathrm{IV}_{2}$ (Guo et al., 2017).

The full length UQCRFS1 is the last subunit to be incorporated into CIII. Previous studies suggested that the tetratricopeptide repeat domain 19 (TTC19) protein collaborates with an inner membrane protease SLP2-PARL-YME1L (SPY) complex to remove UQCRFS1N (residues 1-78 amino acids) from the full length UQCRFS1 after its incorporation into CIII (Bottani et al., 2017; Wai et al., 2016). In the mitochondria of the $\mathrm{Ttc}^{-19^{-/}}$knockout mouse, the native molecular mass of CIII was slightly higher than that of CIII isolated from the wild type animals. This small difference might be the incorporation of two UQCRFS1N segments (which are still parts of the full-length UQCRFS1) into CIII, as opposed to the one UQCRFS1N subunit in the mature CIII. More recently, it was suggested that the CIII core subunits, UQCRC1 and UQCRC2, have the matrix processing peptidase (MPP) activity to cleave the UQCRFS1N peptide after UQCRFS1 is incorporated into the nascent pre-CIII dimer (Fernandez-Vizarra and Zeviani, 2017). On the other hand, UQCRFS1N could inhibit the MPP activity of the UQCRC1- 


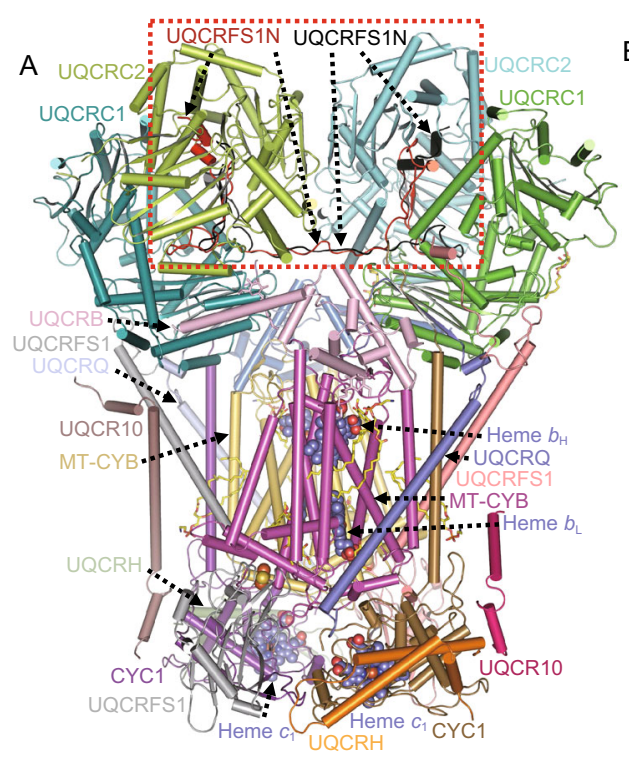

C
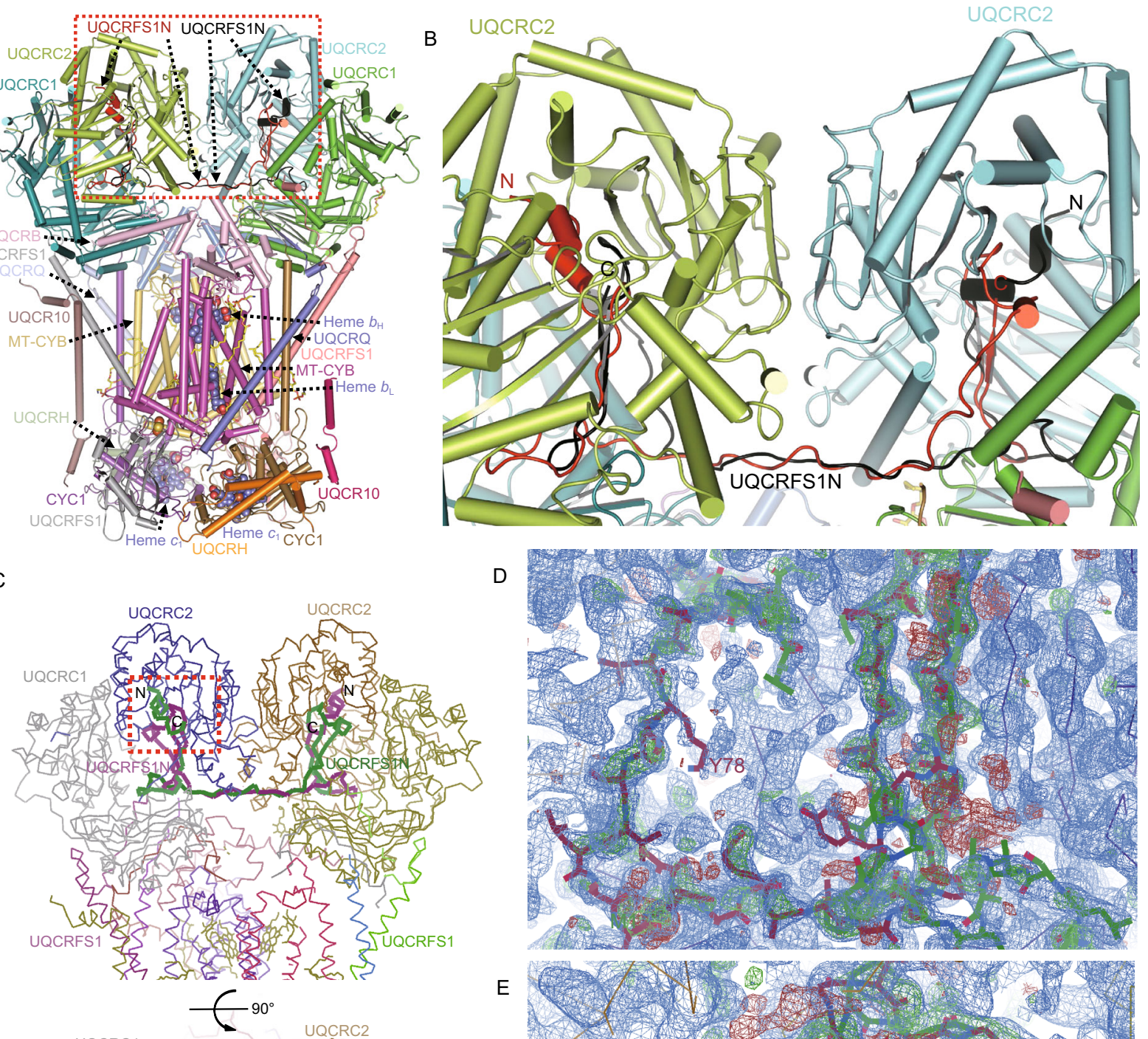

E

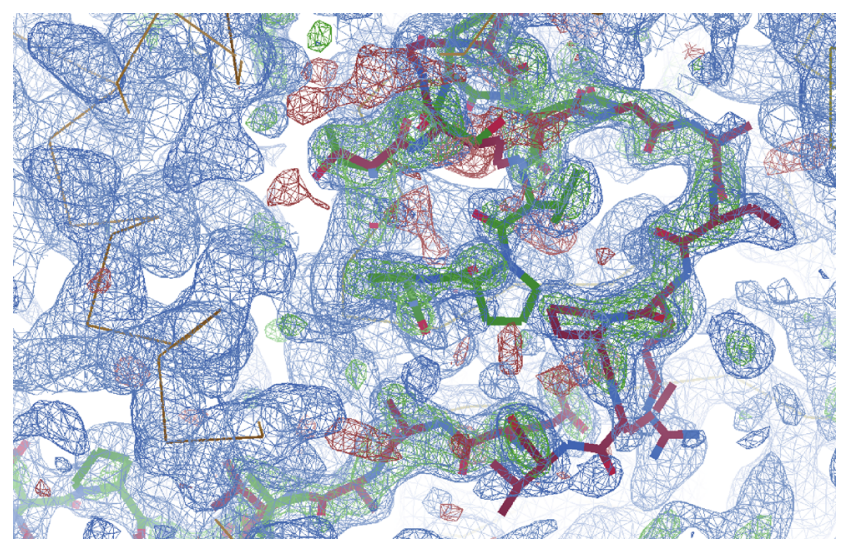


4 Figure 1. UQCRFS1N assembles the 21-subunit CIII. (A) The overall refined structure model of the 21-subunit asymmetric bovine CIII (PDB: 2A06). Different subunits of CIII are labelled and shown in different colors. The two possible orientations of UQCRFS1N are shown in red and black, respectively. (B) Structure of the UQCRFS1N subunit in two possible orientations. $\mathrm{N}$ and $\mathrm{C}$ termini of UQCRFS1N are labelled. This panel is the details of the red dashed frame in (A). (C) The UQCRFS1N structures of bovine CIII (PDB: 2A06). The left panel is viewed along the inner membrane, while the right panel is viewed from the matrix side. UQCRFS1N subunits of two possible orientations are shown in green and purple, respectively. Different subunits of $\mathrm{CIII}$ are labeled and shown in different colors. $\mathrm{N}$ terminal and $\mathrm{C}$ terminal of UQCRFS1 $\mathrm{N}$ are labeled. The red dashed frames indicate two different binding regions of UQCRFS1N in UQCRC2 and UQCRC1. This figure is generated in COOT. (D) The density map of the region indicated by the left red dashed frame in (C). The UQCRFS1N subunits in two switched orientations are shown in sticks and in different colors. Green and blue meshes are mFo-DFc and $2 \mathrm{mFo}-\mathrm{DFc}$, respectively. This figure is generated by COOT. (E) The density map of the region indicated by the right red dashed frame in (C, bottom panel). Shown in the same way as (C, bottom panel). (F) The UQCRFS1N maps of chicken CIII (PDB: 3TGU). Shown in the same way as (C).
UQCRC2 complex in vitro (Deng et al., 2001). Our structures show that one UQCRFS1N molecule can bind to both cavities formed by UQCRC1 and UQCRC2 in both protomers. It is unclear whether UQCRC1-UQCRC2 is indeed involved in the processing of UQCRFS1. More biological experiments are needed to clarify how UQCRFS1 is processed and how CIII is assembled.

In conclusion, after refinement of previous high resolution crystal structures of CIII, we find UQCRFS1N can incorporate into $\mathrm{Clll}$ dimer in two possible orientations, indicating CIII is a 21-subunit asymmetric dimer rather than a 22-subunit homodimer, and both orientations of UQCRFS1N can exist in respirasome, supporting that both protomers of CIII in respirasome are functional and are possible to interact with $\mathrm{Cl}$ to form the megacomplex $\mathrm{I}_{2} \mathrm{II}_{2} \mathrm{IV}_{2}$.

\section{ACKNOWLEDGEMENTS}

We thank the Tsinghua University Branch of China National Center for Protein Sciences (Beijing) for providing the facility support. The computation was completed on the "Explorer 100" cluster system of Tsinghua National Laboratory for Information Science and Technology. This work was supported by funds from the National Key R\&D Program of China (Nos. 2017YFA0504600 and 2016YFA0501100). The National Science Fund for Distinguished Young Scholars

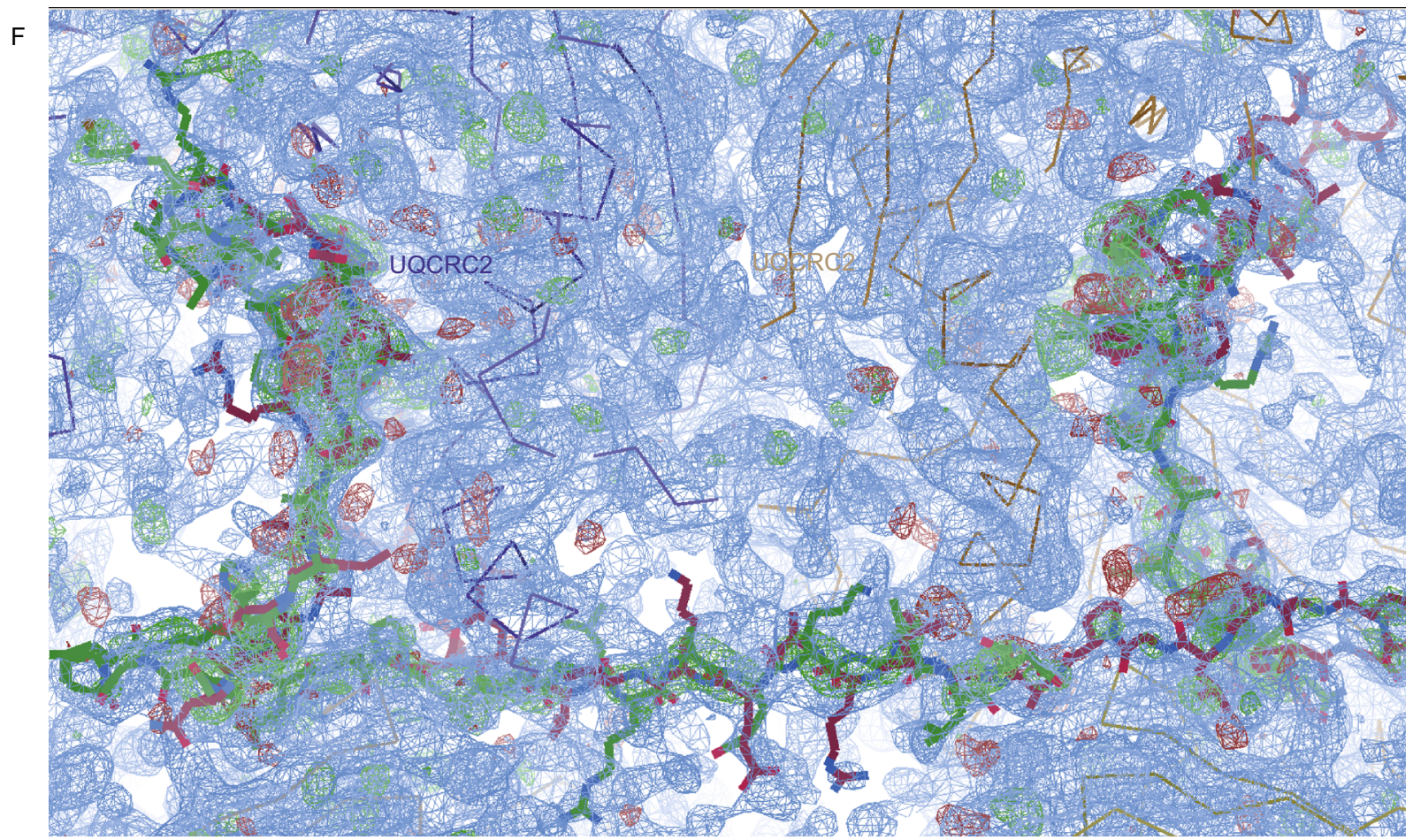

Figure 1. continued. 
(31625008), and the National Natural Science Foundation of China (Grant Nos. 21532004 and 31570733).

\section{AUTHORS CONTRIBUTIONS}

M.Y. conceived, designed and supervised the project, built the model, analyzed the data and wrote the manuscript. S.Z., J.G., T.L. R.G., and M.W. performed all the refinement of Clll structures. All authors discussed the data of the manuscript.

\section{COMPLIANCE WITH ETHICAL STANDARDS}

The authors declare no competing financial interests.

Shuai Zong ${ }^{1}$, Jinke $\mathrm{Gu}^{1}$, Tianya Liu ${ }^{1}$, Runyu Guo ${ }^{1}$, Meng $\mathrm{Wu}^{1}$, Maojun Yang ${ }^{1,2 \bowtie}$

${ }^{1}$ Ministry of Education Key Laboratory of Protein Science, Tsinghua-Peking Joint Center for Life Sciences, Beijing Advanced Innovation Center for Structural Biology, School of Life Sciences, Tsinghua University, Beijing 100084, China

2 School of Pharmacy, Tongji Medical College, Huazhong University of Science and Technology, Wuhan 430030, China

$\triangle$ Correspondence: maojunyang@tsinghua.edu.cn (M. Yang)

\section{OPEN ACCESS}

This article is distributed under the terms of the Creative Commons Attribution 4.0 International License (http://creativecommons.org/ licenses/by/4.0/), which permits unrestricted use, distribution, and reproduction in any medium, provided you give appropriate credit to the original author(s) and the source, provide a link to the Creative Commons license, and indicate if changes were made.

\section{REFERENCES}

Baradaran R, Berrisford JM, Minhas GS, Sazanov LA (2013) Crystal structure of the entire respiratory complex I. Nature 494:443-448

Bottani E, Cerutti R, Harbour ME, Ravaglia S, Dogan SA, Giordano C, Fearnley IM, D'Amati G, Viscomi C, Fernandez-Vizarra E et al (2017) TTC19 plays a husbandry role on UQCRFS1 turnover in the biogenesis of mitochondrial respiratory complex III. Mol Cell 67(96-105):e104
Deng K, Shenoy SK, Tso SC, Yu L, Yu CA (2001) Reconstitution of mitochondrial processing peptidase from the core proteins (subunits I and II) of bovine heart mitochondrial cytochrome bc (1) complex. J Biol Chem 276:6499-6505

Fernandez-Vizarra E, Zeviani M (2015) Nuclear gene mutations as the cause of mitochondrial complex III deficiency. Front Genet 6:134

Fernandez-Vizarra E, Zeviani M (2017) Mitochondrial complex III Rieske Fe-S protein processing and assembly. Cell Cycle 1-25.

Gu J, Wu M, Guo R, Yan K, Lei J, Gao N, Yang M (2016) The architecture of the mammalian respirasome. Nature 537:639-643

Guo R, Gu J, Wu M, Yang M (2016) Amazing structure of respirasome: unveiling the secrets of cell respiration. Protein Cell 7:854-865

Guo R, Zong S, Wu M, Gu J, Yang M (2017) Architecture of human mitochondrial respiratory megacomplex I2III2IV2. Cell 170(12471257):e1212

Hao GF, Wang F, Li H, Zhu XL, Yang WC, Huang LS, Wu JW, Berry EA, Yang GF (2012) Computational discovery of picomolar $Q$ (o) site inhibitors of cytochrome bc1 complex. J Am Chem Soc 134:11168-11176

Huang LS, Cobessi D, Tung EY, Berry EA (2005) Binding of the respiratory chain inhibitor antimycin to the mitochondrial bc1 complex: a new crystal structure reveals an altered intramolecular hydrogen-bonding pattern. J Mol Biol 351:573-597

Schagger H, Pfeiffer K (2000) Supercomplexes in the respiratory chains of yeast and mammalian mitochondria. EMBO J 19:17771783

Vinothkumar KR, Zhu J, Hirst J (2014) Architecture of mammalian respiratory complex I. Nature 515:80-84

Wai T, Saita S, Nolte H, Muller S, Konig T, Richter-Dennerlein R, Sprenger HG, Madrenas J, Muhlmeister M, Brandt U et al (2016) The membrane scaffold SLP2 anchors a proteolytic hub in mitochondria containing PARL and the i-AAA protease YME1L. EMBO Rep 17:1844-1856

Wu M, Gu J, Guo R, Huang Y, Yang M (2016) Structure of mammalian respiratory supercomplex I1III2IV1. Cell 167(15981609):e1510

Xia D, Yu CA, Kim H, Xia JZ, Kachurin AM, Zhang L, Yu L, Deisenhofer J (1997) Crystal structure of the cytochrome bc1 complex from bovine heart mitochondria. Science 277:60-66

Shuai Zong, Jinke Gu, Tianya Liu, Runyu Guo, and Meng Wu have contributed equally to this work. 